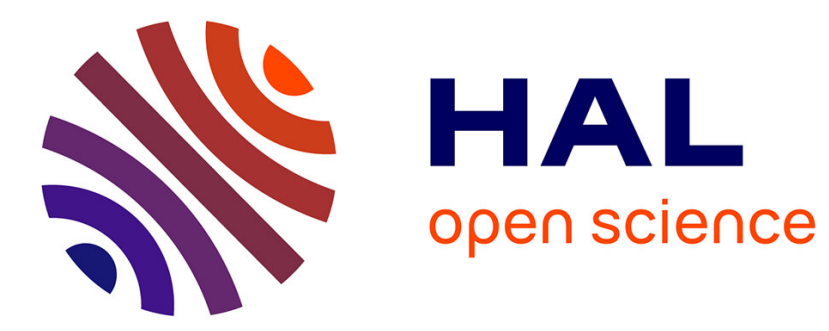

\title{
Comparison of Linear Precoding Schemes for the Massive MIMO Downlink
}

Jakob Hoydis, Stephan ten Brink, Mérouane Debbah

\section{To cite this version:}

Jakob Hoydis, Stephan ten Brink, Mérouane Debbah. Comparison of Linear Precoding Schemes for the Massive MIMO Downlink. IEEE ICC 2012, Jun 2012, Ottawa, Canada. pp.2135 - 2139, 10.1109/ICC.2012.6364360 . hal-00769467

\section{HAL Id: hal-00769467}

https://hal-centralesupelec.archives-ouvertes.fr/hal-00769467

Submitted on 8 Jan 2013

HAL is a multi-disciplinary open access archive for the deposit and dissemination of scientific research documents, whether they are published or not. The documents may come from teaching and research institutions in France or abroad, or from public or private research centers.
L'archive ouverte pluridisciplinaire HAL, est destinée au dépôt et à la diffusion de documents scientifiques de niveau recherche, publiés ou non, émanant des établissements d'enseignement et de recherche français ou étrangers, des laboratoires publics ou privés. 


\title{
Comparison of Linear Precoding Schemes for Downlink Massive MIMO
}

\author{
Jakob Hoydis*§, Stephan ten Brink ${ }^{\ddagger}$, and Mérouane Debbah ${ }^{\S}$ \\ *Department of Telecommunications and ${ }^{\circledR}$ Alcatel-Lucent Chair on Flexible Radio, Supélec, France \\ $\ddagger$ Bell Labs, Alcatel-Lucent, 70435 Stuttgart, Germany \\ \{jakob.hoydis, merouane.debbah\}@supelec.fr \\ stephan.tenbrink@bell-labs.com
}

\begin{abstract}
We consider the downlink of a time-division duplexing (TDD) multicell multiuser MIMO system where the base stations (BSs) are equipped with a very large number of antennas. Assuming channel estimation through uplink pilots, arbitrary antenna correlation and user distributions, we derive approximations of achievable rates with linear precoding techniques, namely eigenbeamforming (BF) and regularized zero-forcing (RZF). The approximations are tight in the large system limit with an infinitely large number of antennas and user terminals (UTs), but match our simulations for realistic system dimensions. We further show that a simple RZF precoding scheme can achieve the same performance as $\mathrm{BF}$ with a significantly reduced number of antennas in both uncorrelated and correlated fading channels.
\end{abstract}

\section{INTRODUCTION}

The use of multiple antennas at the base stations (BSs) is an integral part of future wireless cellular systems [1] as it allows to serve multiple user terminals (UTs) simultaneously on the same resource block and to counter inter- and intracell interference [2]. However, these advantages come at the cost of overhead for the acquisition of channel state information (CSI) at the BSs. In frequency-division duplexing (FDD) systems, this overhead scales linearly with the number of antennas and renders the use of very large antenna arrays essentially impossible [3]. In time-division duplexing (TDD) systems where channel reciprocity can be exploited, the training overhead scales linearly with the number of UTs. Hence, additional antenna elements can be added at no overhead cost to significantly improve the system performance [4].

In [5], the idea of "massive MIMO" is pioneered and several conclusions for a system with an unlimited number of BS antennas are drawn. Theoretically, when the number of antennas grows without bound, the effects of fast fading, imperfect CSI and uncorrelated interference vanish and the system performance is ultimately limited by pilot contamination, resulting from the reuse of the same pilot sequences in adjacent cells [6], [7], [8]. Moreover, the simplest forms of precoding and detection, i.e., eigenbeamforming (BF) and matched filtering (MF), become optimal and the transmit power at BSs and UTs can be made arbitrarily small.

Several subsequent works study the extent to which the above conclusions hold for a large but finite number of antennas and more realistic channel models. In [9], a physical channel model is considered where the number of degrees of freedom the channel provides does not grow with the number of antennas. Here, the main insight is that the performance of massive MIMO might be rather limited by the physical channel conditions than by the number of antennas. In a later paper, the same authors analyze to which extent large antenna arrays allow for transmit power reduction in an uplink singlecell scenario [10]. They conclude that the transmit power can be made inversely proportional to the square root of the number of BS antennas without performance loss. The convergence rate of the performance to its asymptotic limit is studied in [11] and it is observed that generally more antennas are needed to achieve close to optimal performance when the pilot contamination effect is weak. In [12], the authors define and distinguish "massive MIMO" from classical MIMO schemes as a particular operating condition of cellular systems where multiuser interference and noise are dominated by pilot contamination. Whether a system is operated under "massive MIMO conditions" depends consequently on several system parameters, such as the number of BS antennas per UT and the path loss. The authors also study how many more antennas are needed with MF to achieve the same performance as the minimum-mean-square-error (MMSE) detector in the uplink. Finally, [13] proposes a TDD-based system architecture assuming BS cooperation and zero-forcing precoding which achieves massive MIMO spectral efficiencies with one order of magnitude fewer antennas per UT per cell.

In this work, we study the massive MIMO downlink, accounting explicitly for path loss, channel estimation, pilot contamination and arbitrary antenna correlation. The same model was analyzed in [12] for the uplink. We consider a large system limit where the number of BS antennas $N$ and the number of UTs per cell $K$ grow infinitely large at the same speed and derive approximations of achievable rates for different precoding strategies, i.e., BF and regularized zeroforcing (RZF). These approximations are easy to compute and shown by simulations to be accurate for realistic system dimensions. Our results can hence be used for further system optimization, which could not be carried out based on simulations. We further demonstrate that a simple precoding scheme, such as RZF, outperforms BF by far and achieves the same performance with a much smaller number of antennas per UT. Our work contains several novel contributions to the field of large random matrix theory which are of independent interest. 


\section{SYSTEM MODEL}

Consider a multi-cellular system consisting of $L>1$ cells with one BS and $K$ UTs per cell. Each BS is equipped with $N$ antennas; the UTs have a single antenna. The focus of this paper is on the downlink without any form of BS cooperation. The received signal $y_{j m} \in \mathbb{C}$ of the $m$ th UT in the $j$ th cell is given as

$$
y_{j m}=\sqrt{\rho} \sum_{l=1}^{L} \mathbf{h}_{l j m}^{\mathrm{H}} \mathbf{s}_{l}+q_{j m}
$$

where $\mathbf{h}_{l j m} \in \mathbb{C}^{N}$ is the channel vector from BS $l$ to UT $m$ in cell $j, \mathbf{s}_{l} \in \mathbb{C}^{N}$ is the transmit vector of BS $l, q_{j m} \sim$ $\mathcal{C N}(0,1)$ models thermal noise at the receiver and $\rho>0$ is the transmit signal-to-noise ratio (SNR). We assume that the transmit vectors $\mathbf{s}_{l}$ are given as

$$
\mathbf{s}_{l}=\sqrt{\lambda_{l}} \sum_{m=1}^{K} \mathbf{w}_{l m} x_{l m}=\sqrt{\lambda_{l}} \mathbf{W}_{l} \mathbf{x}_{l}
$$

where $\mathbf{W}_{l}=\left[\mathbf{w}_{l 1} \cdots \mathbf{w}_{l K}\right] \in \mathbb{C}^{N \times K}$ is a precoding matrix and $\mathbf{x}_{l}=\left[x_{l 1} \cdots x_{l K}\right]^{\top} \in \mathbb{C}^{K}$ contains the data symbols $x_{l m} \sim \mathcal{C N}(0,1)$ for the $K$ UTs in cell $l$. The normalization factors $\lambda_{l}$ are defined as

$$
\lambda_{l}=\frac{1}{\mathbb{E}\left[\frac{1}{K} \operatorname{tr} \mathbf{W}_{l} \mathbf{W}_{l}^{\mathrm{H}}\right]}
$$

such that the average transmit power per UT is normalized to $\mathbb{E}\left[\frac{\rho}{K} \mathbf{s}_{l}^{\mathrm{H}} \mathbf{s}_{l}\right]=\rho$. We model the channel vectors $\mathbf{h}_{j l k}$ as

$$
\mathbf{h}_{j l k}=\tilde{\mathbf{R}}_{j l k} \mathbf{u}_{j l k}
$$

where $\mathbf{R}_{j l k} \triangleq \tilde{\mathbf{R}}_{j l k} \tilde{\mathbf{R}}_{j l k}^{\mathrm{H}} \in \mathbb{C}^{N \times N}$ are deterministic transmit covariance matrices and $\mathbf{u}_{j l k} \sim \mathcal{C N}\left(\mathbf{0}, \mathbf{I}_{N}\right)$ are fast fading channel vectors. The present channel model is quite general as it allows us to consider a different antenna correlation (including path loss) for each channel vector. This is relevant for large antenna arrays where strong antenna correlation arises from either insufficient antenna spacing or a lack of scattering. In contrast to existing works on massive MIMO (e.g., [13]), the matrices $\mathbf{R}_{j l k}$ do not need to have full rank.

\section{A. Channel estimation}

During an uplink training phase (whose length we ignore in this work), the UTs in each cell transmit orthogonal pilot sequences which allow the BSs to compute estimates $\hat{\mathbf{h}}_{j j k}$ of their local channels $\mathbf{h}_{j j k}$. The same set of orthogonal pilot sequences is reused in every cell so that the channel estimates are corrupted by pilot contamination from adjacent cells [7], [5]. Under these assumptions, BS $j$ estimates the channel vector $\mathbf{h}_{j j k}$ based on the observation

$$
\mathbf{y}_{j k}^{\tau}=\mathbf{h}_{j j k}+\sum_{l \neq j} \mathbf{h}_{j l k}+\frac{1}{\sqrt{\rho_{\tau}}} \mathbf{n}_{j k}
$$

where $\mathbf{n}_{j k} \sim \mathcal{C N}\left(\mathbf{0}, \mathbf{I}_{N}\right)$ and $\rho_{\tau}>0$ is the effective training SNR. The MMSE estimate $\hat{\mathbf{h}}_{j j k}$ of $\mathbf{h}_{j j k}$ is hence given as [14]

$$
\hat{\mathbf{h}}_{j j k}=\mathbf{R}_{j j k} \mathbf{Q}_{j k} \mathbf{y}_{j k}^{\tau}
$$

where

$$
\mathbf{Q}_{j k}=\left(\frac{1}{\rho_{\tau}} \mathbf{I}_{N}+\sum_{l} \mathbf{R}_{j l k}\right)^{-1} .
$$

In order to compute these estimates, the BSs need to have full knowledge of the correlation matrices $\mathbf{R}_{j l k}$. Since the channel vectors are complex Gaussian, we can decompose the channel ${\underset{\tilde{\mathbf{h}}}{j j k}}_{\mathbf{h}_{j j k}}=\hat{\mathbf{h}}_{j j k}+\tilde{\mathbf{h}}_{j j k}$, where $\hat{\mathbf{h}}_{j j k} \sim \mathcal{C N}\left(\mathbf{0}, \boldsymbol{\Phi}_{j j k}\right)$ and $\tilde{\mathbf{h}}_{j j k} \sim \mathcal{C N}\left(\mathbf{0}, \mathbf{R}_{j j k}-\boldsymbol{\Phi}_{j j k}\right)$ is the independent estimation error, and where the matrices $\boldsymbol{\Phi}_{j l k}$ are defined as

$$
\boldsymbol{\Phi}_{j l k}=\mathbf{R}_{j j k} \mathbf{Q}_{j k} \mathbf{R}_{j l k} .
$$

\section{ACHIEVABLE DOWNLINK RATES WITH LINEAR BEAMFORMING}

Since the UTs are not assumed to have any channel estimates, we provide an ergodic achievable rate based on the techniques developed in [6]. To this end, we decompose the received signal $y_{j m}$ as

$$
\begin{aligned}
y_{j m}= & \sqrt{\rho \lambda_{j}} \mathbb{E}\left[\mathbf{h}_{j j m}^{\mathrm{H}} \mathbf{w}_{j m}\right] x_{j m}+\sqrt{\rho \lambda_{j}} \mathbf{h}_{j j m}^{\mathrm{H}} \mathbf{w}_{j m} x_{j m} \\
& -\sqrt{\rho \lambda_{j}} \mathbb{E}\left[\mathbf{h}_{j j m}^{\mathrm{H}} \mathbf{w}_{j m}\right] x_{j m} \\
& +\sum_{(l, k) \neq(j, m)} \sqrt{\rho \lambda_{l}} \mathbf{h}_{l j m}^{\mathrm{H}} \mathbf{w}_{l k} x_{l k}+q_{j m}
\end{aligned}
$$

and assume that the average effective channels $\sqrt{\lambda_{j}} \mathbb{E}\left[\mathbf{h}_{j j m}^{\mathrm{H}} \mathbf{w}_{j m}\right]$ can be perfectly learned at the UTs. Under this assumption, an ergodic achievable rate $R_{j m}$ of UT $m$ in cell $j$ is given as [6, Theorem 1]

$$
R_{j m}=\log _{2}\left(1+\gamma_{j m}\right)
$$

where the associated signal-to-interference-plus-noise ratio (SINR) $\gamma_{j m}$ is defined as ${ }^{1}$

$$
\begin{aligned}
& \gamma_{j m}= \\
& \frac{\lambda_{j}\left|\mathbb{E}\left[\mathbf{h}_{j j m}^{\mathrm{H}} \mathbf{w}_{j m}\right]\right|^{2}}{\frac{1}{\rho}+\lambda_{j} \operatorname{var}\left[\mathbf{h}_{j j m}^{\mathrm{H}} \mathbf{w}_{j m}\right]+\sum_{(l, k) \neq(j, m)} \lambda_{l} \mathbb{E}\left[\left|\mathbf{h}_{l j m}^{\mathrm{H}} \mathbf{w}_{l k}\right|^{2}\right]} .
\end{aligned}
$$

Remark 3.1: Assuming a block-fading channel model with coherence time $T$, one can account for the rate loss due to channel training by considering the net ergodic achievable rate $(1-\tau / T) R_{j m}$ for a given training length $\tau \in[K, T]$ ( $\rho_{\tau}$ would depend also on $\tau$ ). However, the optimization of the training length is not part of this paper and left to future work.

In the sequel, we consider two different linear beamforming techniques: eigenbeamforming $(\mathrm{BF})$ and regularized zero-forcing (RZF). The precoding matrices $\mathbf{W}_{j}$ under both schemes are respectively defined as

$$
\begin{aligned}
\mathbf{W}_{j}^{\mathrm{BF}} \triangleq \hat{\mathbf{H}}_{j j} \\
\mathbf{W}_{j}^{\mathrm{RZF}} \triangleq\left(\hat{\mathbf{H}}_{j j} \hat{\mathbf{H}}_{j j}^{\mathrm{H}}+\mathbf{F}_{j}+N \alpha \mathbf{I}_{N}\right)^{-1} \hat{\mathbf{H}}_{j j}
\end{aligned}
$$

$$
{ }^{1} \operatorname{var}[x] \triangleq \mathbb{E}\left[(x-\mathbb{E}[x])(x-\mathbb{E}[x])^{\mathrm{H}}\right] \text { for some random variable } x \text {. }
$$


where $\hat{\mathbf{H}}_{j j}=\left[\hat{\mathbf{h}}_{j j 1} \cdots \hat{\mathbf{h}}_{j j K}\right] \in \mathbb{C}^{N \times K}, \mathbf{F}_{j} \in \mathbb{C}^{N \times N}$ is a deterministic Hermitian nonnegative definite matrix and $\alpha>0$ is a regularization factor. As the choice of $\mathbf{F}_{j}$ and $\alpha$ is arbitrary, they could be optimized (see e.g., [6, Theorem 6]). However, this is not part of the present work. We denote by $\gamma_{j m}^{\mathrm{MF}}$ and $\gamma_{j m}^{\mathrm{RZF}}$ the SINR under BF and RZF, respectively.

\section{Asymptotic AnALYSis}

In this section, we present our main technical results. All proofs are provided in [15]. As the achievable rates $R_{j m}$ are difficult to compute for finite system dimensions, we consider in this work the large system limit, where $N$ and $K$ grow infinitely large while keeping a finite ratio $K / N$. This is in contrast to [5] which assumes that $K$ remains fixed while $N$ grows without bound. We will retrieve the results of [5] as a special case. The large system limit implicitly assumes that the coherence time of the channel scales linearly with $K$ (to allow for orthogonal pilot sequences of the UTs in a cell). However, as we use the asymptotic analysis only as a tool to provide approximations for finite $N, K$, this does not pose any problem. In the remainder of this section, we will derive approximations $\bar{\gamma}_{j m}$ of the SINR $\gamma_{j m}$ for both BF and RZF, such that

$$
\gamma_{j m}-\bar{\gamma}_{j m} \rightarrow 0
$$

Since the logarithm is a continuous function, (14) implies that

$$
R_{j m}-\log _{2}\left(1+\bar{\gamma}_{j m}\right) \rightarrow 0 .
$$

The results must be understood in the way that, for each given set of system parameters $(N, K)$, we provide a SINR approximation $\bar{\gamma}_{j m}$ which becomes increasingly tight as $N$ and $K$ grow. As we will show in Section $\mathrm{V}$ by simulations, the approximations are very tight even for realistic system dimensions and hence of practical value. Having explicitly computable approximations of achievable rates allows one to compare the impact of different system parameters (antenna correlation, path loss, precoding vectors, etc.) without the need for lengthy Monte Carlo simulations. Our analysis circumvents the problem of computing the expectations in (11) explicitly by providing asymptotic approximations of all terms which are tight in the large system limit. As a nice by-product, our asymptotic SINR approximations are also valid for the case where each UT knows the channels $\hat{\mathbf{h}}_{j l k}$ and even for the case of perfect CSI.

In the sequel, the notation " $N \rightarrow \infty$ " denotes $K, N \rightarrow \infty$ such that $\lim \sup K / N<\infty$. Moreover, we assume that the following conditions hold $\forall j, l, k$ :

A 1: $\lim \sup _{N}\left\|\mathbf{R}_{j l k}\right\|<\infty, \liminf { }_{N} \frac{1}{N} \operatorname{tr} \mathbf{R}_{j l k}>0$.

Our first result provides an asymptotic approximation $\bar{\gamma}_{j m}^{\mathrm{BF}}$ of the SINR with BF $\gamma_{j m}^{\mathrm{BF}}$ :

Theorem 1 (Eigenbeamforming):

$$
\gamma_{j m}^{\mathrm{BF}}-\bar{\gamma}_{j m}^{\mathrm{BF}} \underset{N \rightarrow \infty}{\longrightarrow} 0
$$

where $\bar{\gamma}_{j m}^{\mathrm{BF}}$ is defined as in (16) (top of the next page) and

$$
\bar{\lambda}_{j}=\left(\frac{1}{K} \sum_{k=1}^{K} \frac{1}{N} \operatorname{tr} \boldsymbol{\Phi}_{j j k}\right)^{-1}, \quad 1 \leq j \leq L .
$$

By letting $K / N \rightarrow 0$, we obtain the following corollary which provides the ultimately achievable SINR with infinitely many antennas:

Corollary 1 (BF with an unlimited number of antennas):

$$
\bar{\gamma}_{j m}^{\mathrm{BF}} \underset{N \rightarrow \infty, K / N \rightarrow 0}{\longrightarrow} \frac{\bar{\lambda}_{j}^{\infty} \beta_{j j m}^{2}}{\sum_{l \neq j} \bar{\lambda}_{l}^{\infty}\left|\beta_{l j m}\right|^{2}}
$$

where $\beta_{l j k}=\lim _{N} \frac{1}{N} \operatorname{tr} \boldsymbol{\Phi}_{l j k}$, whenever the limit exists, and $\bar{\lambda}_{j}^{\infty}=\left(\frac{1}{K} \sum_{k=1}^{K} \beta_{j j k}\right)^{-1}$.

Remark 4.1: If the UTs are identically distributed in each cell, the power normalization factors $\bar{\lambda}_{j}^{\infty}$ are equal and our result coincides with [5].

Next we provide an asymptotically tight approximation $\bar{\gamma}_{j m}^{\text {RZF }}$ of the SINR under RZF $\gamma_{j m}^{\mathrm{RZF}}$ :

Theorem 2 (Regularized Zero-Forcing):

$$
\gamma_{j m}^{\mathrm{RZF}}-\bar{\gamma}_{j m}^{\mathrm{RZF}} \underset{N \rightarrow \infty}{\longrightarrow} 0
$$

where $\bar{\gamma}_{j m}^{\text {RZF }}$ is defined as in (17) (top of the next page) and

$$
\begin{aligned}
\bar{\lambda}_{l}= & \frac{K}{N}\left(\frac{1}{N} \operatorname{tr} \mathbf{T}_{l}-\frac{1}{N} \operatorname{tr}\left(\frac{\mathbf{F}_{j}}{N}+\alpha \mathbf{I}_{N}\right) \overline{\mathbf{T}}_{l}^{\prime}\right)^{-1} \\
\vartheta_{l j m}= & \frac{1}{N} \operatorname{tr} \boldsymbol{\Phi}_{l j m} \mathbf{T}_{l} \\
\vartheta_{l j m k}^{\prime}= & \frac{1}{N} \operatorname{tr} \boldsymbol{\Phi}_{l j m} \mathbf{T}_{l k}^{\prime} \\
\mu_{l j m k}= & \frac{1}{N} \operatorname{tr} \mathbf{R}_{l j m} \mathbf{T}_{l k}^{\prime} \\
& -\frac{2 \operatorname{Re}\left(\vartheta_{l j m}^{*} \vartheta_{l j m k}^{\prime}\right)\left(1+\delta_{l m}\right)-\left|\vartheta_{l j m}\right|^{2} \delta_{l m k}^{\prime}}{\left(1+\delta_{l m}\right)^{2}}
\end{aligned}
$$

and where

- $\mathbf{T}_{l}=\mathbf{T}(\alpha)$ and $\boldsymbol{\delta}_{l}=\left[\delta_{l 1} \cdots \delta_{l K}\right]^{\top}=\boldsymbol{\delta}(\alpha)$ are given by Theorem 3 for $\mathbf{S}=\mathbf{F}_{j} / N, \mathbf{D}=\mathbf{I}_{N}$ and $\mathbf{R}_{k}=\boldsymbol{\Phi}_{l l k} \forall k$,

- $\overline{\mathbf{T}}_{l}^{\prime}=\mathbf{T}^{\prime}(\alpha)$ is given by Theorem 4 for $\mathbf{S}=\mathbf{F}_{j} / N$, $\boldsymbol{\Theta}=\mathbf{I}_{N}, \mathbf{D}=\mathbf{I}_{N}$ and $\mathbf{R}_{k}=\boldsymbol{\Phi}_{l l k} \forall k$,

- $\mathbf{T}_{l k}^{\prime}=\mathbf{T}^{\prime}(\alpha), \boldsymbol{\delta}_{l k}^{\prime}=\left[\delta_{l 1 k}^{\prime} \cdots \delta_{l K k}^{\prime}\right]^{\mathbf{T}}=\boldsymbol{\delta}^{\prime}(\alpha)$ are given by Theorem 4 for $\mathbf{S}=\mathbf{F}_{j} / N, \mathbf{\Theta}=\boldsymbol{\Phi}_{l l k}, \mathbf{D}=\mathbf{I}_{N}$ and $\mathbf{R}_{k}=\boldsymbol{\Phi}_{l l k} \forall k$.

Theorems 3 and 4 can be found in the Appendix.

Remark 4.2: The expression of $\bar{\gamma}_{j m}^{\text {RZF }}$ can be significantly simplified under a less general channel model, e.g., no antenna correlation, Wyner-type models with a simple path loss factor, no estimation noise $\left(\rho_{\tau} \rightarrow \infty\right)$. Due to the page restriction, we only provide the most general form.

For an infinitely large number of antennas per UT, we get the following SINR with RZF: 


$$
\begin{gathered}
\bar{\gamma}_{j m}^{\mathrm{BF}}=\frac{\bar{\lambda}_{j}\left(\frac{1}{N} \operatorname{tr} \boldsymbol{\Phi}_{j j m}\right)^{2}}{\frac{1}{N \rho}+\frac{1}{N} \sum_{l, k} \bar{\lambda}_{l} \frac{1}{N} \operatorname{tr} \mathbf{R}_{l j m} \boldsymbol{\Phi}_{l l k}+\sum_{l \neq j} \bar{\lambda}_{j}\left|\frac{1}{N} \operatorname{tr} \boldsymbol{\Phi}_{l j m}\right|^{2}} \\
\bar{\gamma}_{j m}^{\mathrm{RZF}}=\frac{\bar{\lambda}_{j} \delta_{j m}^{2}}{\frac{1}{N \rho}\left(1+\delta_{j m}\right)^{2}+\frac{1}{N} \sum_{l, k} \bar{\lambda}_{l}\left(\frac{1+\delta_{j m}}{1+\delta_{l k}}\right)^{2} \mu_{l j m k}+\sum_{l \neq j} \bar{\lambda}_{l}\left(\frac{1+\delta_{j m}}{1+\delta_{l m}}\right)^{2}\left|\vartheta_{l j m}\right|^{2}}
\end{gathered}
$$

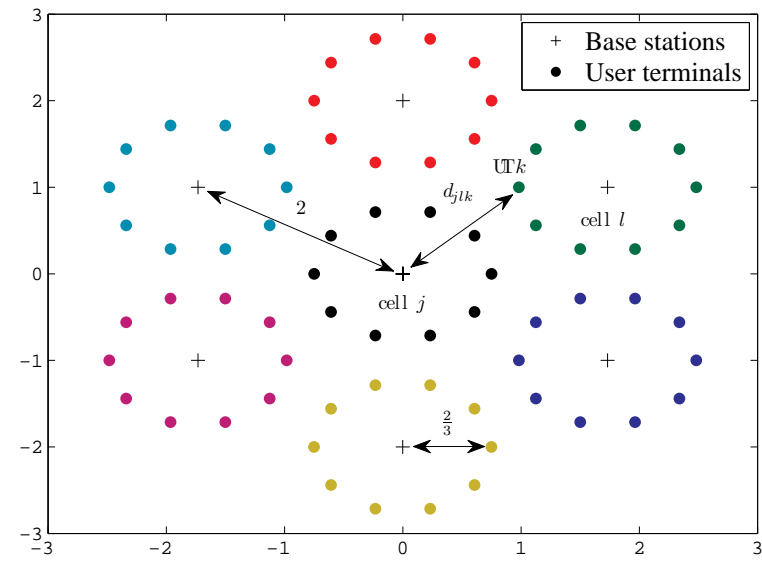

Fig. 1. 7-cell hexagonal system layout. The distance between two adjacent cells is normalized to 2 . There are $K=10$ UTs uniformly distributed on a circle of radius $2 / 3$ around each $\mathrm{BS}$.

\section{Corollary 2 ( $R Z F$ with an unlimited number of antennas):}

$$
\bar{\gamma}_{j m}^{\mathrm{RZF}} \underset{N \rightarrow \infty, K / N \rightarrow 0}{\longrightarrow} \frac{\bar{\lambda}_{j}^{\infty} \beta_{j j m}^{2}}{\sum_{l \neq j}\left(\frac{\alpha+\beta_{j j m}}{\alpha+\beta_{l l m}}\right)^{2} \bar{\lambda}_{l}^{\infty}\left|\beta_{l j m}\right|^{2}}
$$

where $\beta_{l j k}=\lim _{N} \frac{1}{N} \operatorname{tr} \boldsymbol{\Phi}_{l j k}$, whenever the limit exists, and $\bar{\lambda}_{j}^{\infty}=\left(\frac{1}{K} \sum_{k=1}^{K} \frac{\beta_{j j k}}{\left(\alpha+\beta_{j j k}\right)^{2}}\right)^{-1}$.

Remark 4.3: Even for $\bar{\lambda}_{j}^{\infty}=\bar{\lambda}_{k}^{\infty} \forall k$, the SINR under $\mathrm{RZF}$ and $\mathrm{BF}$ with an unlimited number of antennas are not necessarily identical (cf. Corollary 1). This is because the received interference power depends on the correlation matrices $\boldsymbol{\Phi}_{l l m}$ of the UTs in the neighboring cells. This is in contrast to the uplink [12] where the matched filter and the MMSE detector have the same asymptotic performance.

\section{NumericAl EXAMPLES}

In order to validate the accuracy of the asymptotic rate approximations for finite $N, K$, we consider an hexagonal system with $L=7$ cells as shown in Fig. 1. The inner cell radius is normalized to one and we assume a distance-based path loss model with path loss exponent $\beta=3.7$. To allow for reproducibility of our results, we distribute $K=10$ UTs uniformly on a circle of radius $2 / 3$ around each BS and do not consider shadowing. We further assume a training SNR $\rho_{\tau}=6 \mathrm{~dB}$ and transmit SNR $\rho=10 \mathrm{~dB}$. For RZF, we use a regularization factor $\alpha=1 / \rho$ and $\mathbf{F}_{j}=0$ (this is similar to

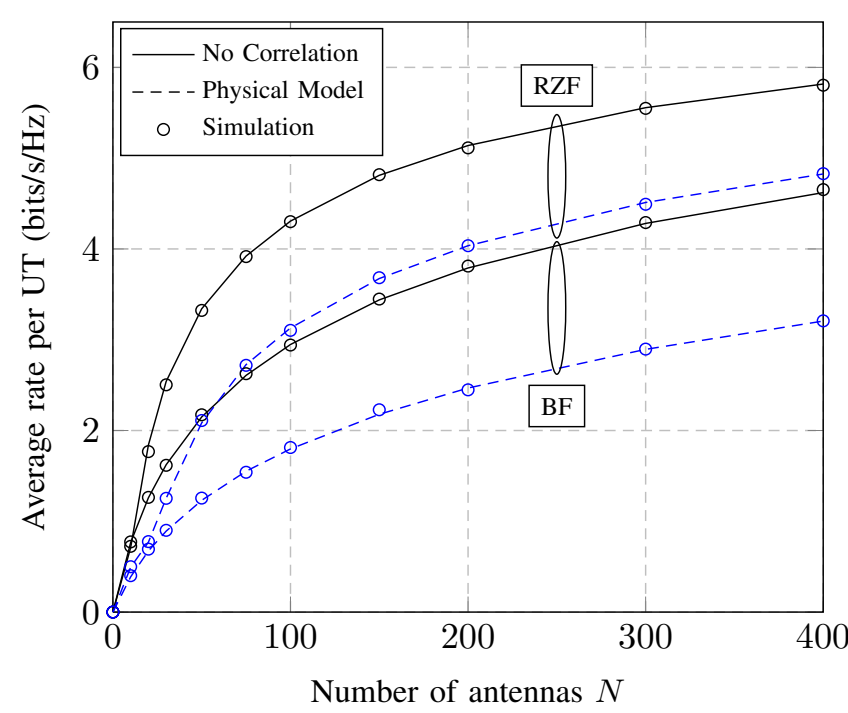

Fig. 2. Average per-user rate versus number of antennas $N$ for the RZF and $\mathrm{BF}$ precoders. Solid and dashed lines depict the asymptotic approximations, markers the simulation results.

MMSE precoding). Average rates are then calculated for the UTs in the center cell.

First, we consider a simple channel model without antenna correlation, i.e., $\tilde{\mathbf{R}}_{j l k}=d_{j l k}^{-\beta / 2} \mathbf{I}_{N}$, where $d_{j l k}$ is the distance between BS $j$ and the $k$ th UTs in cell $l$ (cf. (4)). For an unlimited number of antennas, BF achieves an ultimate rate of $7.2 \mathrm{bits} / \mathrm{s} / \mathrm{Hz}$ while RZF achieves $7.08 \mathrm{bits} / \mathrm{s} / \mathrm{Hz}$. In Fig. 2, we show the achievable rates under both precoding techniques and their approximations by Theorems 1 and 2 as a function of the number of antennas $N$. Both results match very well, even for small $N$. We can observe that RZF achieves significant performance gains over $\mathrm{BF}$ as it reduces multiuser interference. In the present setting, RZF allows us to reduce the number of antennas roughly by a factor 4 to achieve BFperformance. Nevertheless, even for $N=400$ both precoders are far away from their ultimate performance limits.

Next, we consider a physical channel model with a fixed number of dimensions $P$ as in [9]. For a uniform linear array, the matrices $\tilde{\mathbf{R}}_{j l k}=d_{j l k}^{-\beta / 2}\left[\mathbf{A} \mathbf{0}_{N \times N-P}\right]$, where $\mathbf{A}=$ $\left[\mathbf{a}\left(\phi_{1}\right) \cdots \mathbf{a}\left(\phi_{P}\right)\right] \in \mathbb{C}^{N \times P}$ is composed of the "steering vectors" $\mathbf{a}(\phi) \in \mathbb{C}^{N}$ defined as $(\mathbf{i}=\sqrt{-1})$

$$
\mathbf{a}(\phi)=\frac{1}{\sqrt{P}}\left[1, e^{-\mathbf{i} 2 \pi c \sin (\phi)}, \ldots, e^{-\mathbf{i} 2 \pi c(N-1) \sin (\phi)}\right]^{\top}
$$


where $c$ is the antenna spacing in multiples of the wavelength and $\phi_{p}=-\pi / 2+(p-1) \pi / P, p=1, \ldots, P$, are the uniformly distributed angles of transmission. We assume that the physical dimensions $P$ scale with the number of antennas as $P=N / 2$ and $c=0.3$. Since $\frac{1}{N} \operatorname{tr} \mathbf{A} \mathbf{A}^{\mathrm{H}}=1$, the ultimately achievable rates under this channel model are equal to those of the channel model without antenna correlation. For comparison, we depict in Fig. 2 also the achievable rates and their approximations for this channel model. Interestingly, while the shapes of the curves for both precoders are similar to those without antenna correlation, it becomes clear that a lower rank of the correlation matrices (or fewer channel degrees of freedom) severely degrades the performance.

\section{CONCLUSions}

We have studied the downlink performance of linear precoders in multicell multiuser TDD systems with a large number of BS antennas. Assuming a large system limit, we have derived approximations of achievable rates for BF and RZF, which are shown to be tight for realistic system dimensions. Our results indicate that with RZF, the number of antennas can be significantly reduced to achieve the same performance as the simple BF scheme. Since massive MIMO TDD-systems are a promising future cellular network architecture, it seems necessary to verify the theoretical performance predictions by channel measurements and prototypes.

\section{APPENDIX}

Theorem 3 ([16, Theorem 1]): Let $\mathbf{D} \in \mathbb{C}^{N \times N}$ and $\mathbf{S} \in$ $\mathbb{C}^{N \times N}$ be Hermitian nonnegative definite matrices and let $\mathbf{H} \in \mathbb{C}^{N \times K}$ be a random matrix with columns $\mathbf{h}_{k}=$ $\frac{1}{\sqrt{N}} \mathbf{R}_{k}^{\frac{1}{2}} \mathbf{u}_{k}$, where $\mathbf{u}_{k} \in \mathbb{C}^{N}$ are random vectors of i.i.d. elements with zero mean, unit variance and finite 8th order moment, and $\mathbf{R}_{k} \in \mathbb{C}^{N \times N}$ are deterministic covariance matrices. Assume that $\mathbf{D}$ and the matrices $\mathbf{R}_{k}, k=1, \ldots, K$, have uniformly bounded spectral norms (with respect to $N$ ). Let $N, K \rightarrow \infty$, such that $\limsup \frac{K}{N}<\infty$. Then, for any $\rho>0$,

$$
\frac{1}{N} \operatorname{tr} \mathbf{D}\left(\mathbf{H H}^{\mathrm{H}}+\mathbf{S}+\rho \mathbf{I}_{N}\right)^{-1}-\frac{1}{N} \operatorname{tr} \mathbf{D T}(\rho) \stackrel{\text { a.s. }}{\longrightarrow} 0
$$

where $\mathbf{T}(\rho) \in \mathbb{C}^{N \times N}$ is defined as

$$
\mathbf{T}(\rho)=\left(\frac{1}{N} \sum_{k=1}^{K} \frac{\mathbf{R}_{k}}{1+\delta_{k}(\rho)}+\mathbf{S}+\rho \mathbf{I}_{N}\right)^{-1}
$$

and $\boldsymbol{\delta}(\rho)=\left[\delta_{1}(\rho) \cdots \delta_{K}(\rho)\right]^{\top}$ is the unique solution to

$$
\delta_{k}(\rho)=\frac{1}{N} \operatorname{tr} \mathbf{R}_{k} \mathbf{T}(\rho), \quad k=1, \ldots, K
$$

satisfying $\delta_{k}(\rho) \geq 0 \forall k$.

Theorem 4: Let $\Theta \in \mathbb{C}^{N \times N}$ be a Hermitian nonnegative definite matrix with uniformly bounded spectral norm. Under the same conditions as in Theorem 3,

$$
\begin{aligned}
\frac{1}{N} \operatorname{tr} & \mathbf{D}\left(\mathbf{H H}^{\mathrm{H}}+\mathbf{S}+\rho \mathbf{I}_{N}\right)^{-1} \boldsymbol{\Theta}\left(\mathbf{H H}^{\mathrm{H}}+\mathbf{S}+\rho \mathbf{I}_{N}\right)^{-1} \\
& -\frac{1}{N} \operatorname{tr} \mathbf{D} \mathbf{T}^{\prime}(\rho) \stackrel{\text { a.s. }}{\longrightarrow} 0
\end{aligned}
$$

where $\mathbf{T}^{\prime}(\rho) \in \mathbb{C}^{N \times N}$ is defined as

$$
\mathbf{T}^{\prime}(\rho)=\mathbf{T}(\rho) \boldsymbol{\Theta} \mathbf{T}(\rho)+\mathbf{T}(\rho) \frac{1}{N} \sum_{k=1}^{K} \frac{\mathbf{R}_{k} \delta_{k}^{\prime}(\rho)}{\left(1+\delta_{k}(\rho)\right)^{2}} \mathbf{T}(\rho)
$$

with $\mathbf{T}(\rho)$ and $\delta_{k}(\rho)$ as defined in Theorem 3 and $\boldsymbol{\delta}^{\prime}(\rho)=$ $\left[\delta_{1}^{\prime}(\rho) \cdots \delta_{K}^{\prime}(\rho)\right]^{\top}$ given by

$$
\begin{aligned}
\boldsymbol{\delta}^{\prime}(\rho) & =\left(\mathbf{I}_{K}-\mathbf{J}(\rho)\right)^{-1} \mathbf{v}(\rho) \\
{[\mathbf{J}(\rho)]_{k l} } & =\frac{\frac{1}{N} \operatorname{tr} \mathbf{R}_{k} \mathbf{T}(\rho) \mathbf{R}_{l} \mathbf{T}(\rho)}{N\left(1+\delta_{l}(\rho)\right)^{2}} \\
{[\mathbf{v}(\rho)]_{k} } & =\frac{1}{N} \operatorname{tr} \mathbf{R}_{k} \mathbf{T}(\rho) \Theta \mathbf{T}(\rho)
\end{aligned}
$$

where $\mathbf{J}(\rho) \in \mathbb{C}^{K \times K}$ and $\mathbf{v}(\rho) \in \mathbb{C}^{K}$.

\section{REFERENCES}

[1] S. Sesia, M. Baker, and I. Toufik, LTE: The UMTS Long Term Evolution: From Theory to Practice. Wiley-Blackwell, Jul. 2011.

[2] D. Gesbert, M. Kountouris, R. W. Heath, C.-B. Chae, and T. Salzer, "Shifting the MIMO paradigm," IEEE Signal Process. Mag., vol. 24, no. 5, pp. 36-46, Sep. 2007.

[3] G. Caire, N. Jindal, M. Kobayashi, and N. Ravindran, "Multiuser MIMO achievable rates with downlink training and channel state feedback," IEEE Trans. Inf. Theory, vol. 56, no. 6, pp. 2845-2866, Jun. 2010.

[4] T. L. Marzetta, "How much training is required for multiuser MIMO?" in Proc. Asilomar Conf. on Signals, Systems and Computers, UrbanaChampaign, Illinois, US, Sep. 2006, pp. 359-363.

[5] _ - "Noncooperative cellular wireless with unlimited numbers of base station antennas," IEEE Trans. Wireless Commun., vol. 9, no. 11, pp. 3590-3600, Nov. 2010.

[6] J. Jose, A. Ashikhmin, T. Marzetta, and S. Vishwanath, "Pilot contamination and precoding in multi-cell TDD systems," IEEE Trans. Wireless Commun., vol. 10, no. 8, pp. 2640-2651, Aug. 2011.

[7] J. Jose, A. Ashikhmin, T. L. Marzetta, and S. Vishwanath, "Pilot contamination problem in multi-cell TDD systems," in Proc. IEEE Int'l Symp. Inform. Theory, Jul. 2009, pp. 2184-2188.

[8] K. Appaiah, A. Ashikhmin, and T. L. Marzetta, "Pilot contamination reduction in multi-user TDD systems," in Proc. IEEE Int'l Conf. Commun., May 2010, pp. 1-5.

[9] H. Q. Ngo, E. G. Larsson, and T. L. Marzetta, "Analysis of the pilot contamination effect in very large multicell multiuser MIMO systems for physical channel models," in Proc. IEEE Int'l Conf. on Acoustics, Speech and Signal Processing, Prague, Czech Repulic, May 2011, pp. 3464-3467.

[10] — , "Uplink power efficiency of multiuser MIMO with very large antenna arrays," in Proc. Allerton Conf. on Communication, Control, and Computing, Urbana-Champaign, Illinois, US, Sep. 2011.

[11] B. Gopalakrishnan and N. Jindal, "An analysis of pilot contamination on multi-user MIMO cellular systems with many antennas," in Proc. IEEE Int'l Workshop in Signal Processing Advances in Wireless Communications, San Francisco, CA, US, Jun. 2011.

[12] J. Hoydis, S. ten Brink, and M. Debbah, "Massive MIMO: How many antennas do we need?" in Proc. Allerton Conf. on Communication, Control, and Computing, Urbana-Champaign, Illinois, US, Sep. 2011.

[13] H. Huh, G. Caire, H. C. Papadopoulos, and S. A. Ramprashad, "Achieving massive MIMO spectral efficiency with a not-so-large number of antennas," 2011, submitted to IEEE Trans. on Wireless Commun. [Online]. Available: http://arxiv.org/abs/1107.3862

[14] S. Verdú, Multiuser Detection. Cambridge University Press, 1998.

[15] J. Hoydis, S. ten Brink, and M. Debbah, "Massive MIMO in the UL/DL of cellular networks: How many antennas do we need?" 2012, submitted, available upon request.

[16] S. Wagner, R. Couillet, M. Debbah, and D. T. M. Slock, "Large system analysis of linear precoding in MISO broadcast channels with limited feedback," IEEE Trans. Inf. Theory, 2012, to appear. [Online]. Available: http://arxiv.org/pdf/0906.3682v3 